\title{
Dropped head syndrome and bent spine syndrome: two separate clinical entities or different manifestations of axial myopathy?
}

\author{
W G H Oerlemans, $M$ de Visser
}

\begin{abstract}
Four elderly women are reported on with myopathies manifesting with isolated or predominant involvement of the paraspinal musculature. In three, the neck extensors were affected foremost, leading to "dropped head syndrome". In one, weakness of the thoracic paraspinal muscles caused "bent spine syndrome". It is suggested that these clinically distinct syndromes are caused by a primary tardive myopathic condition predominantly affecting the entire axial musculature.

(F Neurol Neurosurg Psychiatry 1998;65:258-259)
\end{abstract}

Keywords: dropped head syndrome; bent spine syndrome; axial myopathy

Over the past 10 years a few reports have appeared, describing mostly elderly patients, presenting with relatively restricted weakness of the paraspinal musculature, either at the cervical or at the thoracic level, of unknown origin. ${ }^{1-11}$ We report four additional cases: three with "dropped head syndrome" and one with "bent spine syndrome". We hypothesise that these neuromuscular conditions might have a similar aetiology.

Neurology, Westeinde

Ziekenhuis, The

Hague, The

Netherlands

W G H Oerlemans

Department of

Neurology, Academic

Medical Center,

Amsterdam, The

Netherlands

$M$ de Visser

Correspondence to:

Dr WGH Oerlemans,

Department of Neurology,

Westeinde Ziekenhuis,

Lijnbaan 32, PO Box 432,

$2501 \mathrm{CK}$, The Hague,

The Netherlands.

Telephone 0031703302000 fax 00-31-70-3809459.

Received 21 July 1997 and in final revised form 27 January 1998

Accepted 30 January 1998

\section{Case reports}

CASE 1

A 65 year old woman with cervical spinal stenosis established by MRI complained of the inability to prevent her head from dropping on her chest (figure). Neurological examination disclosed isolated neck extensor weakness but no other relevant abnormalities. Laboratory studies including serum creatine kinase $(\mathrm{CK})$ and calcium were normal. Antibodies against acetylcholine receptors (AchRs) were absent. EMG disclosed short duration, low amplitude motor unit action potentials (MUAPs) of paraspinal and sternocleidomastoid muscles. CT of the skeletal musculature showed fatty changes of neck extensors, thoracic and lumbar paraspinal muscles, and of the hamstrings. A biopsy specimen from the trapezius muscle showed non-specific changes including an increase in the number of fibres with internal nuclei, fibre splitting, clumps of pycnotic nuclei, and moth-eaten and targetoid fibres.

A neck brace was prescribed and follow up for over one year has not shown any progress of the disease.

CASE 2

An 82 year old woman with a history of breast carcinoma in 1973, cervical spondylarthosis, and a lumbar spinal stenosis, presented with slowly progressive neck extensor weakness over a period of three months. Neurological examination disclosed paresis of the neck extensors and extensor plantar responses. Laboratory studies including CK and AchRs were normal. EMG disclosed signs of reinnervation, and repetitive nerve stimulation was normal. CT showed fatty changes of the paraspinal muscles at the cervical, thoracic, and lumbar level, and atrophy of the thigh muscles. A biopsy specimen taken from a cervical paraspinal muscle showed marked variation in the diameter of muscle fibres, fibre splitting, an increase in the number of fibres with internal nuclei, some necrotic fibres with a few surronding monuclear cells, moth-eaten fibres, an occasional ragged red and regenerating fibre, and one fibre with rimmed vacuoles.

Since 1994 there has been slight progression with subsequent involvement of the shoulder girdle muscles.

CASE 3

A 69 year old woman presented with neck extensor weakness after having had the "flu". Neurological examination showed weakness of the neck musculature, extensors more than flexors, and slight paresis of the proximal musculature of the limbs. Sensation was undisturbed and reflexes were normal.

Serum CK was increased up to twice the upper limit of normal; AchRs were negative and EMG showed myopathic changes. Repetitive nerve stimulation was negative and so was a single fibre EMG. Two biopsy specimens taken from the quadriceps and the deltoid muscles, disclosed myopathic abnormalities, type 2 fibre atrophy, minimal inflammatory cells, and some atrophic, angular sized muscle fibres, staining darkly with non-specific esterase. CT showed lower attenuation of the 


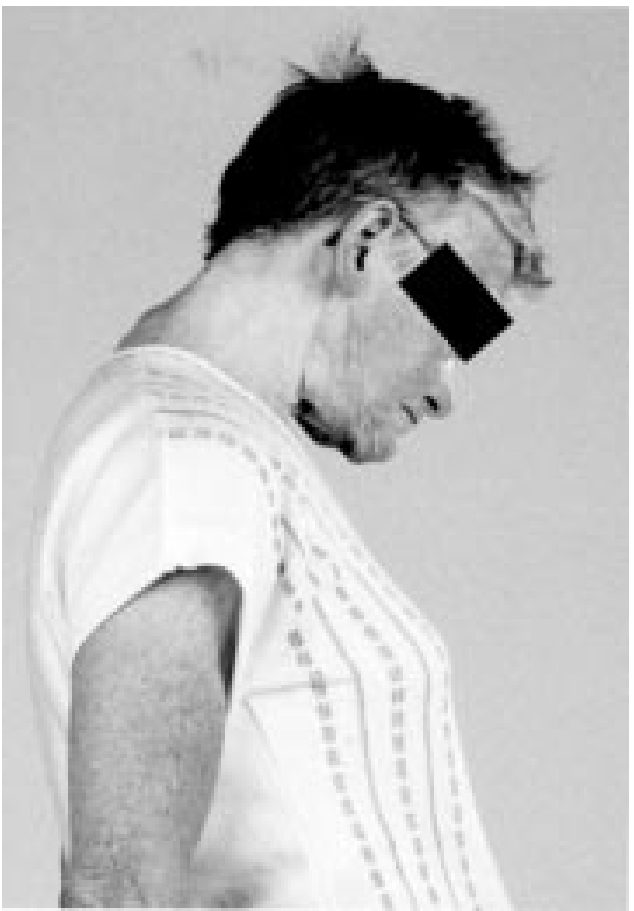

Case 1; attempt to keep her head in an upright position. (With permission.)

paraspinal muscles, mostly at the thoracic level. In addition, some fatty changes in the hamstrings, gluteal, quadriceps, and calf musculature were seen.

Her condition remained stable over a period of a year.

CASE 4

A 77 year old woman known to have congestive heart failure was evaluated for over one year for a progressive gait disorder which prevented her from keeping an upright position while standing and walking. Neurological examination disclosed paresis of the thoracic erectores spinae and a mild paresis of the proximal arm musculature. Laboratory investigations including $\mathrm{CK}$ and AchRs were normal; EMG disclosed brief MUAPs in the axial musculature. No fasiculations were found. CT showed fatty changes of the paraspinal muscles. In the thigh and lower leg muscles fatty replacement of muscles had also taken place. Histopathological examination of an affected muscle could not be performed because the patient died unexpectedly of congestive heart failure.

\section{Discussion}

Our four patients had an isolated or predominant weakness of axial musculature. This presentation led to an extensive differential diagnosis including myasthenia gravis, amyotrophic lateral sclerosis, and idiopathic inflammatory myopathies representing the most often found and relevant neuromuscular disorders.

Clinical presentation and data from ancillary investigations were not suggestive of any of these diseases. Therefore, the diagnosis "dropped head syndrome" and "bent spine syndrome" were made, merely on clinical grounds. Our EMG and histopathological findings showed myopathic or neurogenic abnormalities, or a mixture of both and did not essentially differ from those described by others. ${ }^{1-4}{ }^{9-11}$ CT showed replacement of muscles by fatty tissue. In all four patients CT abnormalities were not limited to the level of clinical involvement but showed fatty changes in all paraspinal muscles. Other skeletal muscles were affected as well, although subclinically and to a lesser extent. Several authors have hypothesised on the pathogenesis of these syndromes. A monophasic inflammatory process restricted to the paraspinal musculature has been suggested. ${ }^{3411}$ We found some necrotic and regenerating muscle fibres associated with a few mononuclear cells in one of our patients as did Serratrice et al in one case of "'bent spine syndrome" and Jaster et al in their case of "dropped head syndrome". ${ }^{41}$ However, overt signs of inflammation were not present in our patients.

An alternative explanation is the susceptibility to injury of paraspinal musculature by kyphotic postural changes and age dependent loss of tissue elasticity. ${ }^{3}$ Histopathological findings in paraspinal muscles of patients with lumbar disc herniation or lumbar stenosis are not essentially different from those of patients suffering from "bent spine syndrome", which corroborates this hypothesis. ${ }^{6}$

More likely, we suggest both "dropped head syndrome" and "bent spine syndrome" may be considered to be late manifestations of a primary tardive unclassified myopathy, predominantly affecting axial musculature. Reports of heriditary cases of both syndromes seem to support this notion. ${ }^{49}$ It remains as yet unexplained why a myopathy affecting all paraspinal musculature eventually leads to two distinct clinical syndromes.

1 Suarez GA, Kelly JJ. The dropped head syndrome. Neurology 1992;42:1625-7.

2 Lange DJ, Fettell MR, Lovelace RE, et al. The floppy head syndrome [abstract]. Ann Neurol 1986;20:133.

3 Katz JS, Wolfe GI, Burns DK, et al. Isolated neck extensor myopathy: a common cause of dropped head syndrome. Neurology 1996;46:917-21, and 1997;48:552-3.

4 Serratrice G, Pouget J, Pellisier JF. Bent spine syndrome. $\mathcal{f}$ Neurol Neurosurg Psychiatry 1996;60:51-4.

5 Bleck TP. The dropped head syndrome [correspondence]. Neurology 1993;43:846.

6 Delisle MB, Laroche M, Dupont $\mathrm{H}$, et al. Morphological analyses of paraspinal mucles: comparison of progressive lumbar kyphosis (camptocormia) and narrowing of lumbar canal by disc protrusions. Neuromusc Disord 1993:5/6:57982.

7 Laroche M, Delisle MB. La camptocormie primitive est une myopathie para-vertébrale. Rev Rhum Engl Ed 1994;61: 481-4.

8 Poullin P, Daumen-Legre V, Serratrice G. La camptocormie du sujet agé: myopathie ou dystonie musculaire? Rev Rhum Engl Ed 1993;60:159-61.

9 Waclawik AJ, Lotz BP, Salamat SM, et al. Myopathic dropped head syndrome. An expanding clinico-pathologic spectrum. Neurology (Suppl) 1997;48:445.

10 Waclawik AJ, Lotz BP, Beinlich BR, et al. Dropped head syndrome [letter]. Neurology 1997;48:550-1.

11 Jaster JH, Bertorini TE, Swims MP, et al. Dropped head syndrome [letter]. Neurology 1997;48:552. 